\title{
Dissecting aneurysms of posterior cerebral artery: clinical presentation, angiographic findings, treatment, and outcome
}

\author{
Muhammad A. Taqi ', Marc A. Lazzaro' ${ }^{1}$, Dhruvil J. Pandya ${ }^{1}$, Aamir Badruddin' ${ }^{1}$ and Osama O. Zaidat ${ }^{1,2,3 *}$ \\ 1 Department of Neurology, Medical College of Wisconsin, Milwaukee/Froedtert Hospital and Children Hospital of Wisconsin, Wisconsin, MI, USA \\ ${ }^{2}$ Department of Neurosurgery, Medical College of Wisconsin, Milwaukee/Froedtert Hospital and Children Hospital of Wisconsin, Wisconsin, MI, USA \\ ${ }^{3}$ Department of Radiology, Medical College of Wisconsin, Milwaukee/Froedtert Hospital and Children Hospital of Wisconsin, Wisconsin, MI, USA
}

\section{Edited by:}

David S. Liebeskind, University of California Los Angeles, USA

Reviewed by:

Weihai Xu, Peking Union Medical College Hospital, Chinese Academy of Medical Sciences, China Ronen Leker, Hadassah University Hospital, Israel

*Correspondence:

Osama O. Zaidat, Neurointerventional Program, Medical College of Wisconsin and Froedtert Hospital West, 9200 W, Wisconsin Avenue, Milwaukee, WI 53226, USA.

e-mail: szaidat@mcw.edu

\begin{abstract}
Background:The dissecting posterior cerebral artery (PCA) aneurysms are very rare. These aneurysms pose significant treatment challenge and need careful evaluation to formulate an optimal treatment plan in case of ruptured or un-ruptured presentations. Methods: Retrospective review of a prospectively collected data. Results: Seven patients with dissecting aneurysms of the PCA were identified. Six out of seven presented with subarachnoid hemorrhage (SAH) and one with ischemic stroke. Three out of seven were treated with endovascular coil embolization without sacrifice of the parent artery and the rest had parent artery occlusion (PAO) with coil embolization. None of the patients developed new neurological deficits post-procedure. Aneurysm re-occurred in two patients that were treated without PAO. Conclusion: Endovascular treatment of the dissecting PCA aneurysm is safe and feasible. It can be performed with or without PAO. Recurrence is more common without PAO and close follow-up is warranted.
\end{abstract}

Keywords: PCA aneurysm, dissecting aneurysm, coiling, parent artery occlusion, endovascular, blow out aneurysm, subarachnoid hemorrhage, posterior cerebral artery

\section{INTRODUCTION}

Aneurysms arising from the posterior cerebral artery (PCA) are very rare and comprise about $0.26-1 \%$ of the reported aneurysms cases (Drake and Amacher, 1969). Most of the reported PCA aneurysms are saccular (Hamada et al., 2005). Dissecting PCA aneurysms are less commonly encountered. Dissecting aneurysms of the PCA can be post traumatic or spontaneous. Treatment with coil embolization for these aneurysms in the context of subarachnoid hemorrhage (SAH) is very challenging. To reconstruct the artery without sacrificing PCA requires use of stent; which may be limited by the inability to administer antiplatelet therapy in acutely ruptured aneurysm. Number of the previously reported cases confirmed this dilemma; hence some of aneurysms were left untreated and followed with observation only. Others were treated with endovascular coiling and fewer cases with open surgical approach (Berger and Wilson, 1984; Pozzati et al., 1991; Lazinski et al., 2000; Ciceri et al., 2001; Kiazawa et al., 2001; Hamada et al., 2005; Vilela and Guolao, 2006; Nistri et al., 2007; Renard and Milhaud, 2007; Lv et al., 2009; Oran et al., 2009; Maillo et al., 1991; Hallacq et al., 2002). One of the series reported occipital artery to PCA bypass after endovascular parent artery occlusion (PAO; Chang et al., 2010).

Endovascular therapy using stent-assisted coiling or overlapping stents versus permanent PCA coils occlusion may be considered. The optimal approach may vary according to the anatomy and morphological features of the aneurysm. We present case series of seven patients of PCA dissecting aneurysms that were treated with endovascular therapy. Clinical and radiological presentation, technique, and follow-up data are presented. Discussion and review of the literature is provided. The anatomical divisions of PCA are based on Zeal's classification (Zeal and Rhoton, 1978).

\section{MATERIALS AND METHODS}

The prospective neurointerventional database was reviewed for all cerebral aneurysm coil embolization that were performed at our institution from July 2005 to February 2011. Cases with PCA aneurysm with an angiographic appearance of dissecting aneurysm as judged by the author's consensus were included. Rest of the PCA aneurysms were excluded from the study. Since patients were enrolled from the database of interventional procedures, only symptomatic patients that received treatment were included. Per hospital policy all patients with cerebral aneurysm are treated with endovascular approach within $24 \mathrm{~h}$ of presentation therefore none of the patients were treated surgically. Demographic, clinical and radiological presentation, technical details, peri-procedural complications, and follow-up data was collected on all patients. Outcomes included subsequent need for additional endovascular therapy, and long-term clinical and angiographic results. Descriptive statistics are presented.

\section{CASE SERIES}

All procedures were performed with patients under general anesthesia. A trans-femoral arterial approach was used in all patients. Per protocol, patients without SAH were systemically heparinized to maintain an activated clotting time (ACT) level of 250-300 s. 
Images were obtained with biplane projections as well as a threedimensional rotational digital subtraction angiogram. Working views were obtained after review of the 3-D rotational angiogram. When technically possible to advance the wire safely into the distal PCA segment and track the balloon, hemodynamic evaluation was performed by balloon test occlusion proximal to the planned sacrificing segment with Hyperglide balloon (ev3 Neurovascular, Irvine, CA, USA). This required bilateral femoral artery access and performing carotid cerebral angiography to visualize collaterals. This is an angiographic selective temporary PCA balloon occlusion test.

Special attention was given in evaluating collateral supply and aneurysm proximity to P1 segment perforators. Final angiographic runs were performed prior to completion of each case to evaluate aneurysm residual, parent artery patency, and thrombotic or dissection complications. If parent artery occlusion was performed, then treatment included post-procedure blood pressure augmentation with a goal of increasing the mean arterial pressure by $20-30 \%$ from baseline for $24-72 \mathrm{~h}$ duration using the clinical examination to guide the goal and duration. Patients were examined by the interventional neurologist post-procedure and neurointensivist within $24 \mathrm{~h}$ after procedure.

The treatment approach aimed at preserving or sacrificing the artery was considered after:

(1) Studying the collateral circulation from the middle cerebral artery, anterior cerebral artery and internal carotid artery (ICA) into the affected PCA with or without balloon test occlusion.

(2) Studying the location of the dissecting aneurysm and it's relation to the $\mathrm{P} 1$ and its perforators.

A total of seven patients with dissecting aneurysms of the PCA were identified. Table 1 summarizes the baseline characteristics of the patients, treatment, and follow-ups. The mean age was 37 years \pm 20 (range 5-62 years). Five patients were female, six $(86 \%)$ were Caucasians and one $(14 \%)$ was Hispanic. One patient
(14\%) was a smoker and had known hypertension. No history of recent or remote trauma was identified in any of the patients. Mean duration of clinical follow-up was 22.5 months (11-43 months).

The majority of the patients $(86 \%)$ presented with SAH and only $1(14 \%)$ patient presented with ischemic stroke, most likely related to partial thrombosis of the aneurysm with or without distal clot embolization. Of the six patients, who presented with $\mathrm{SAH}$, three had focal neurological deficits corresponding to the PCA territory. In two of the six ruptured patients; vomiting and coughing were identified as a potential trigger for rupture of the aneurysm.

The aneurysms were located in the P2 segment in four (57\%) patients; while two (29\%) patients had P2/P3 segment aneurysms and one $(14 \%)$ had a P3 segment aneurysm. In five out of seven $(71 \%)$ patients, a large posterior communicating artery (P-Comm) was noted ipsilateral to the aneurysm. The maximum diameter ranged from 5.5 to $28 \mathrm{~mm}$. In six (86\%) patients aneurysms were found on the left side.

All of the patients were treated with an endovascular treatment approach. In four out of seven (57\%) patients, the parent PCA with aneurysm was sacrificed with coil embolization of the aneurysm followed by proximal parent artery occlusion. In one of these patients; delayed parent artery occlusion was performed. This patient initially had stent-assisted coiling; but developed additional growth of the aneurysm and the parent PCA artery had to be sacrificed distal to the thalamic perforators. Half of these patients (two out of four) underwent a balloon occlusion test showing good collateral supply from the anterior circulation before obliterating the PCA. None of the patients developed new symptomatic stroke or new neurological deficits related to the artery sacrificed. The neurological deficit was defined by any increase in the NIHSS post-procedure documented by an independent neurointensivist and interventional neurologist examination that were not blinded to the procedure. The balloon test occlusion was used when feasible by the anatomy of the PCA. If tracking the balloon seemed difficult or sacrifice was the only option for treatment, balloon occlusion was avoided to prevent unnecessary risk.

Table 1| This table summarizes the detailed demographic, presentation, and treatment outcome of the study patients (Nistri et al., 2007).

\begin{tabular}{|c|c|c|c|c|c|c|c|c|c|c|c|}
\hline Pts & Age & Sex & PRS & FND & $\begin{array}{l}\text { Aneurysm } \\
\text { location }\end{array}$ & $\begin{array}{l}\text { Size } \\
(\mathrm{max}, \mathrm{mm})\end{array}$ & Treatment & Event & $\begin{array}{l}\text { Clinical FU } \\
\text { (months) }\end{array}$ & $\begin{array}{l}\text { Radiographic } \\
\text { FU (months) }\end{array}$ & ADR \\
\hline 1 & 23 & $\mathrm{~F}$ & $\mathrm{SAH}$ & LHO & RP2 & 10.5 & $\begin{array}{l}\text { Stent and GDC, } \\
\text { later PAO }\end{array}$ & Thrombus* & 11 & 3 & $\begin{array}{l}\text { New sac } \\
\text { required PAO }\end{array}$ \\
\hline 2 & 48 & $\mathrm{~F}$ & $\mathrm{SAH}$ & NONE & RP2 & 5.5 & Stent and GDC & None & 43 & 43 & None \\
\hline 3 & 5 & $\mathrm{~F}$ & SAH & NONE & LP2/P3 & 28 & $\mathrm{PAO}, \mathrm{GDC}$ & None & 19 & 19 & None \\
\hline 4 & 45 & $F$ & SAH & $\mathrm{RHP}$ & LP2 & 12.5 & PAO, GDC & None & 20 & 13 & None \\
\hline 6 & 25 & M & SAH & $\mathrm{RHP}$ & LP3 & 11 & $\mathrm{PAO}, \mathrm{GDC}$ & None & 10 & - & None \\
\hline 7 & 54 & $\mathrm{~F}$ & SAH & NONE & LP2/P3 & 8 & Stent and GDC & None & 11 & 3 & None \\
\hline
\end{tabular}

m, male; f, female; PRS, presentation; SAH, subarachnoid hemorrhage; IS, ischemic stroke; LHO, left hemianopia; RHP, right hemiparesis; RP, right PCA; LP, left PCA; GDC, Guglielmi detachable coils; PAO, parent artery occlusion; FU, follow-up; APR, additional procedure required.

*Thrombus resolved after intra-arterial Abciximab without any clinical sequel. 
Three $(43 \%)$ patients were ultimately treated with coiling of the aneurysm without occluding the artery and had good results at the end of the procedure. Stent-assisted coiling was used in two of these three patients. In patient \# 7, a stent was placed to protect the superior division of PCA and the inferior division was sacrificed. All patients that received stent were given pre-op or intra-op antiplatelets (Figure 1).

Procedural complication was limited to one patient with asymptomatic thrombus developing at a stent that was successfully treated with intra-arterial abciximab during the procedure. None of the patient developed new or worsening of their clinical symptoms immediately after the procedure as evaluated by change in NIHSS.

\section{DISCUSSION}

We present a series of dissecting PCA aneurysms from a single center that were treated with endovascular techniques. We describe the clinical and angiographic presentation, treatment technique, and outcomes. To our knowledge only few cases of dissecting PCA aneurysms have been described in the literature with the largest series by Lv et al. (2009). In our case series, we have described the clinical presentations and the treatment approach that may help in deciding the optimal endovascular technique and the long-term follow-up of patients with ruptured or unruptured dissecting PCA aneurysms. Our study carries all the drawbacks of a retrospective review.

The exact definition of dissecting aneurysms is not described in the literature. They are mostly reported based on the author's consensus of their angiographic appearance that has been described as "pearl and string" or "blow out." The natural history of these aneurysms is also not well known. In few case reports patients were followed without any surgical or endovascular intervention and had no complications. The true incidence of risk of initial bleed or re-bleed cannot be ascertained.

The most common presentation in our case series was $\mathrm{SAH}$ (six out of seven) secondary to aneurysmal rupture presenting with headache and focal neurological deficits corresponding to the vascular territory of the PCA. Only one patient presented with ischemic stroke without SAH. Ischemic stroke presentation of the dissecting PCA aneurysm is infrequent in this series in line with other reported case series. Of the cases that are reported in literature 54\% presented with SAH, 25\% with focal neurological deficits without SAH and in $21 \%$ aneurysms were discovered incidentally (Berger and Wilson, 1984; Pozzati et al., 1991; Lazinski et al., 2000; Ciceri et al., 2001; Kiazawa et al., 2001; Hamada et al., 2005; Vilela and Guolao, 2006; Nistri et al., 2007; Renard and Milhaud, 2007; Lv et al., 2009; Oran et al., 2009; Chang et al., 2010; Maillo et al., 1991; Hallacq et al., 2002).

Most of the patients were of younger age group suggesting that these aneurysms have different etiology than a traditional saccular aneurysm. Only one patient had typical risk factors for aneurysm including hypertension and smoking. Various etiologies are suggested including infectious (syphilis, mycotic), migraine, cystic medial necrosis, fibromuscular dysplasia, homocysteinuria, mixed connective tissue disease, and trauma (Hamada et al., 2005). There was no significant recent or remote trauma in any patient, although two of the patients had a coughing or sneezing episode before developing the headache, supporting the hypothesis of predisposition to dissection. One of the patients who presented with SAH had multiple spontaneous vessels dissection including one vertebral artery and one ICA in addition to the PCA. Almost all the aneurysm occurred in the region of $\mathrm{P} 2 / \mathrm{P} 3$ segment, this particular part of the PCA traverses across the tentorium cerebri coursing supra-tentorialy. Stress on the vessel wall along the edges of tentorium is one possible theory to explain the development of the dissecting PCA aneurysm (Drake et al., 1996). Concomitant vasculopathies like Moya Moya or AVM are reported to be present in some cases of intracranial dissecting aneurysms suggesting their development is both flow and development related. Berger and Wilson (1984) in their review of dissecting intracranial aneurysm discussed the difference between the intracranial and extra-cranial dissections. The extra-cranial dissections develop between the media and adventitia layers of vessel wall while the intracranial dissections are mostly present between the intimal and media layers and are surrounded by normal adventitia. The reason for this difference is not known but suggests that a small

Images before endovascular treatment

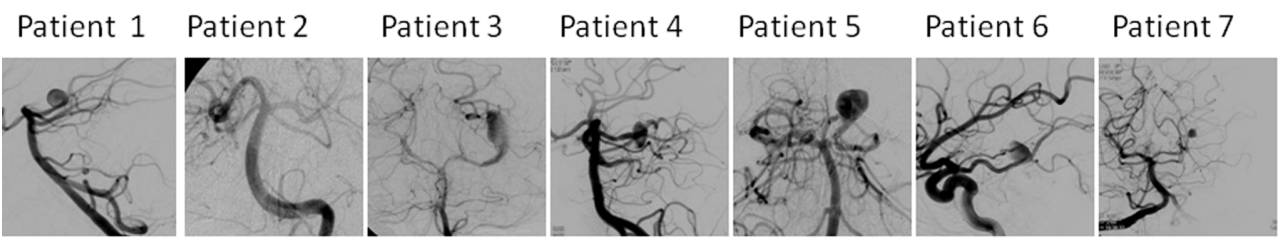

Images after endovascular treatment

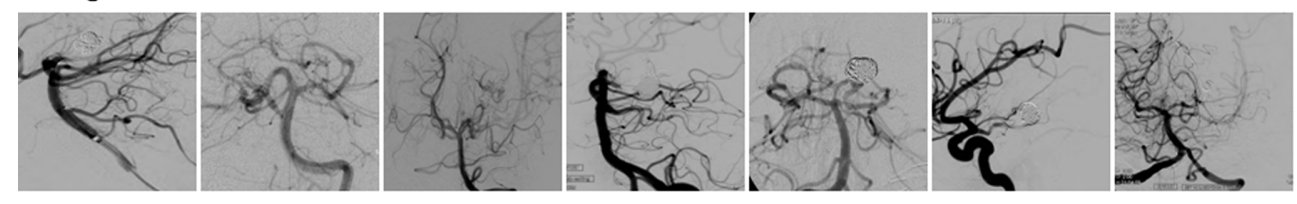

FIGURE 1 | 
Table 2 | Previously reported PCA dissecting aneurysms.

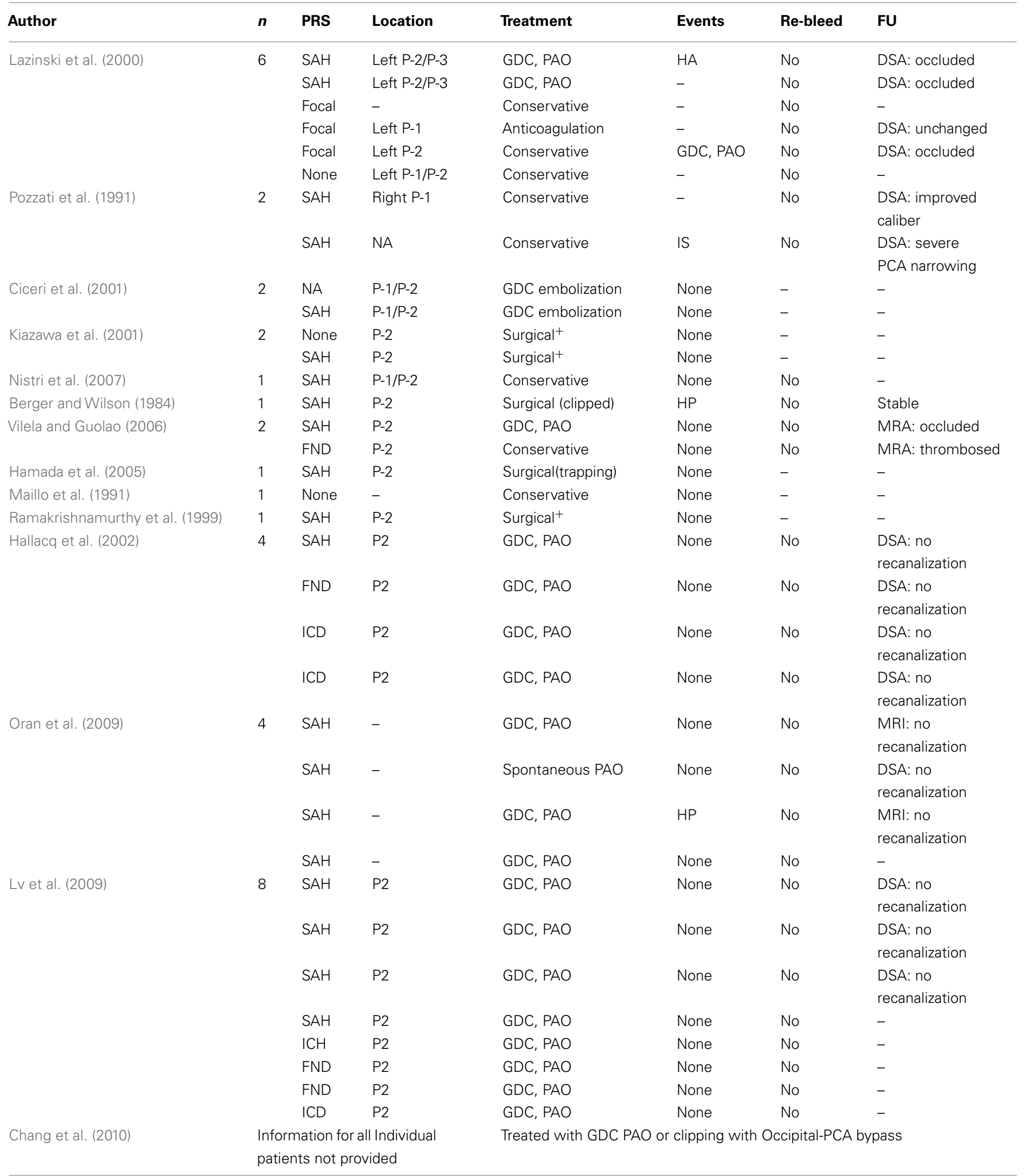

GDC, Guglielmi detachable coils; PAO, parent artery occlusion; SAH, subarachnoid hemorrhage; HA, headache; IS, ischemic stroke; HP, hemiparesis; RHP, right hemiparesis; P, PCA; FND, focal neurological deficits; ICD, incidental; ${ }^{+}$proximal clipping with vessel occlusion. 
tear in the intima, especially at the branching points can be a trigger to intracranial vascular dissections and subsequent aneurysm malformation. We noted that the majority of our patients had an enlarged P-comm, whether this contribute to high flow in the PCA territory and had role in the development of the aneurysm is unknown. Other authors have contributed these aneurysms to high flow states that in some instances is related to associated AVM's (Ciceri et al., 2001).

Although treatment of dissecting aneurysms without obliteration of parent artery has been described for aneurysms other than PCA (Lempert et al., 1998), rarely this approach has been done for dissecting PCA aneurysm. We attempted this approach on four of the patients without sacrificing the parent artery. Neuroform ${ }^{\text {TM }}$ (Boston Scientific, Natick, MA, USA) stents were used in three patients $(1,2$, and 7$)$ when crossing the aneurysm was felt to be technically feasible in order to attempt the stent-assisted coiling. In one of the patients; the PCA was supplying the dissected and occluded ICA via the P-comm and the P1 had to be preserved. The fourth patient had a very large blowout aneurysm with ability to reconstruct the artery with complex shape coils only without the need of stent.

In one case series of open surgical clipping, the aneurysm was wrapped to avoid closure of the artery. No other case series attempted in preserving the artery. Chang et al. presented 14 cases of PAO followed by occipital to PCA bypass. This was associated with significant procedural morbidity and caution was advised using this approach (Chang et al., 2010).

It seems feasible to save the PCA with stent-assisted coiling if potential deficits with sacrifice are of concerns especially in a young patient that could be deprive of driving. However, the durability and safety of artery saving technique cannot be ascertained with this small series. Even in our experience of four cases that were initially had no parent artery occlusion, no re-bleeding occurred, however two of them required retreatment (50\%). First one, later required occlusion of the artery due to regrowth and expansion of the aneurysm, and the second one required recoiling.

\section{REFERENCES}

Berger, M. S., and Wilson, C. B. (1984). Intracranial dissecting aneurysms of the posterior circulation: report of six cases and review of the literature. J. Neurosurg. 61, 882-894.

Chang, S. W., Abla, A. A., Kakarla, U. K., Sauvageau, E., Dashti, S. R., Nakaji, P., Zabramski, J. M., Albuquerque, F. C., McDougall, C. G., and Spetzler, R. F. (2010). Treatment of distal posterior cerebral artery aneurysms: a critical appraisal of the occipital artery-to-posterior cerebral artery bypass. Neurosurgery 67, 16-26.

Ciceri, E. F., Klucznik, R. P., Grossman, R. G., Rose, J. E., and Mawad, M. E. (2001). Aneurysms of posterior cerebral artery: classification and endovascular treatment. AJNR Am. J. Neuroradiol. 22, 27-34.

Drake, C. G., and Amacher, A. L. (1969). Aneurysm of the posterior

It is likely that the definitive endovascular approach to treat these aneurysms is to occlude the parent artery if the aneurysm is distal to the P2 segment. However, it is feasible and technically possible to treat without occluding the artery, if a large deficit is expected from such occlusion, or progression of thrombus to the basilar tip is of a concern. A PCA balloon test occlusion may predict deficits due to artery sacrifice; however, technical difficulty in tracking the balloon must be weighed against the benefit of the test occlusion. Only few of the reported case series described using a PCA balloon occlusion test (Hallacq et al., 2002).

In our case series the final and ultimate treatment was parent artery occlusion in four out of seven (one initially had stentassisted coiling and later complete occlusion was required), coiling only in one case and stent-assisted coiling two out of seven cases. In our cases treated with parent artery occlusion (four out of seven) none of them developed new neurological deficit following the procedure or on discharge. Although this is retrospective study and a neurological deficit is defined by a change in NIHSS. It does not encompass extensive battery of testing that can be performed for temporal and occipital lobes function. Previously reported case series had five post-procedure complications resulting in ischemic strokes in the territory of PCA, two of them developed hemiparesis. The series with occipital to PCA bypass was associated with significant complications from the bypass procedure that included epidural hematoma, occipital infarct/edema or angiographic failure of bypass (Chang et al., 2010). In all these cases the parent artery was sacrificed either by surgical approach or endovascular technique. (Table 2 summarizes the previously reported case series).

\section{CONCLUSION}

Endovascular therapy for the treatment of dissecting aneurysms of the PCA is safe and effective. Angiographic recurrence is more common among patients that are treated without parent artery occlusion and therefore close follow-up is indicated in such patients.

cerebral artery aneurysms: report of eleven cases. J. Clin. Neurosci. 8, 23-26.

Lazinski, D., Willinsky, R. A., TerBrugge, K., and Montanera, W. (2000). Dissecting aneurysms of the posterior cerebral artery: angioarchitecture and a review of the literature. Neuroradiology 42, 128-133.

Lempert, T. E., Halbach, V. V., Higashida, R. T., Dowd, C. F., Urwin, R. W., Balousek, P. A., and Hieshima, G. B. (1998). Endovascular treatment of pseudoaneurysms with electrolytically detachable coils. AJNR Am. J. Neuroradiol. 19, 907-911.

Lv, X., Li, Y, Jiang, C., Yang, X., and Wu, Z. (2009). Parent vessel occlusion for P2 dissecting aneurysms of the posterior cerebral artery. Surg. Neurol. 71, 319-325.

Maillo, A., Diaz, P., and Morales, F. (1991). Dissecting aneurysm of the posterior cerebral artery: spontaneous resolution. Neurosurgery 29, 291-294.

Nistri, M., Perrini, P., Lorenzo, N., Cellerini, M., Villari, N., and Mascalchi, M. (2007). Third nerve palsy heralding dissecting aneurysm of posterior cerebral artery: digital subtraction angiography and magnetic resonance appearance. J. Neurol. Neurosurg. Psychiatr. 78, 197-198.

Oran, I., Cinar, C., Yagci, B., Tarhan, S., Kiroglu, Y., and Serter, S. (2009). Ruptured dissecting aneurysms arising from non-vertebral arteries of the posterior circulation: endovascular treatment perspective. Diagn. Interv. Radiol. 15, 159-165.

Pozzati, E., Padovani, R., Fabrizi, A., Sabattini, L., and Gaist, G. (1991). Benign arterial dissections of the posterior circulation. J. Neurosurg. 75, 69-72. 
Ramakrishnamurthy, T. V., Purohit, A. K., Sundaram, C., Ramamurti , and Rajender, Y. (1999). Distal calcarine fusiform aneurysm: a case report and review of literature. 47, 318-320.

Renard, D., and Milhaud, D. (2007). Dissecting aneurysm of the posterior cerebral artery. $N$. Engl. J. Med. 357, 24.

Vilela, P., and Guolao, A. (2006). Pediatric dissecting posterior cerebral aneurysms: report of two cases and review of the literature. Neuroradiology 48, 541-548.

Zeal, A. A., and Rhoton, A. L. (1978). Microsurgical anatomy of the posterior cerebral artery. $J$. Neurosurg. 48, 534-559.

Conflict of Interest Statement: The authors declare that the research was conducted in the absence of any commercial or financial relationships that could be construed as a potential conflict of interest.

Received: 26 January 2011; accepted: 27 May 2011; published online: 24 June 2011.

Citation: Taqi MA, Lazzaro MA, Pandya $D J$, Badruddin $A$ and Zaidat $O O$ (2011) Dissecting aneurysms of posterior cerebral artery: clinical presentation, angiographic findings, treatment, and outcome. Front. Neur. 2:38. doi: 10.3389/fneur.2011.00038
This article was submitted to Frontiers in Endovascular and Interventional Neurology, a specialty of Frontiers in Neurology.

Copyright (C) 2011 Taqi, Lazzaro, Pandya, Badruddin and Zaidat. This is an openaccess article subject to a non-exclusive license between the authors and Frontiers Media SA, which permits use, distribution and reproduction in other forums, provided the original authors and source are credited and other Frontiers conditions are complied with. 